\title{
Psychotropic Drug-Related Fall Incidents in Nursing Home Residents Living in the Eastern Part of The Netherlands
}

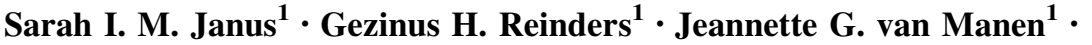 \\ Sytse U. Zuidema ${ }^{2} \cdot$ Maarten J. IJzerman $^{1}$
}

Published online: 8 April 2017

(C) The Author(s) 2017. This article is an open access publication

\begin{abstract}
Background Older people are more susceptible to falls than younger people. Therefore, as the Dutch population ages, the total number of falls and costs associated with them will rise. The use of psychotropic drugs is associated with an increased risk of falling. To create tailored fallprevention programmes, information on the magnitude of the association between fall incidents and specific psychotropic drugs or drug classes is needed.

Objective The goal of this study was to delineate the associations between fall incidents and specific psychotropic drugs or drug classes.

Methods In this retrospective cohort study, electronic patient records, medication records and fall incident reports were collected for 1415 residents receiving somatic or psychogeriatric care in 22 nursing homes in the eastern part of the Netherlands from May 2012 until March 2015. Using a Cox proportional hazards model, we analysed the magnitude of the association between psychotropic drugs and the risk of falling for users and non-users of the psychotropic drugs or drug classes.

Results Antipsychotics (adjusted hazard ratio [aHR] 1.49; 95\% confidence interval $[\mathrm{CI}] 1.12-2.00$ ) and hypnotics and sedatives (aHR 1.51; 95\% CI 1.13-2.02) increase the risk of falling. There was no difference between the risk incurred by typical and atypical antipsychotics. However,
\end{abstract}

Sarah I. M. Janus

s.i.m.janus@utwente.nl

1 Department of Health Technology and Services Research, University of Twente, Enschede, The Netherlands

2 Department of General Practice and Elderly Care Medicine, University of Groningen, University Medical Centre Groningen, Groningen, The Netherlands within these groups, there were differences between the most commonly prescribed drugs: haloperidol and quetiapine were seen to have an association with falls, whereas pipamperone and risperidone were not.

Conclusions The results suggest falls may be associated with individual drugs rather than drug classes. Within the drug classes, clear differences are evident between individual drugs. Future fall-prevention programmes should highlight the differential risks involved with the use of specific psychotropic drugs, and doctors should take the fall risk into account when choosing specific drugs.

\section{Key Points}

Falls within the nursing home population may be associated with individual drugs rather than drug classes.

Differences exist between individual drugs within drug classes.

\section{Introduction}

Over $40 \%$ of nursing home residents experience a least one fall each year [1]. A fall is hereby defined as 'an event that leads to a person coming to rest on the ground or other lower level' [2]. Because the population is aging, and older people have a higher risk of falling, the number of falls is likely to increase. Injuries resulting from falls are prominent among the causes of disability in older people [3]. With an expected increase in age-related incidents such as falls, a rise in associated costs can also be expected [3]. 
While falls are often considered accidents, it has been shown that the incidence of falls significantly differs from a Poisson distribution, implying that falls are causally connected with external factors [5].

Factors that influence the risk of falling (besides age) include state of health $[4,5]$ and drug use. The former is difficult to influence, whereas the latter can be influenced by changing medication prescriptions. For instance, polypharmacy, defined as the simultaneous use of more than five drugs, increases the risk of adverse events such as falls $[4,6,7]$. In the Netherlands, people aged $>75$ years use five times as many prescription drugs as the average citizen, and they use these drugs predominantly chronically [8].

Some drugs are more harmful than others when included in polypharmacy. The Screening Tool of Older Person's Prescriptions (STOPP) lists the potentially inappropriate medications (PIMs) for older people [9], including several classes of psychotropic drugs. In several studies, these drugs were the most prevalent PIMs.

An association between the increased risk of falling in older people and the use of psychotropic drugs was previously reported in the meta-analysis of three systematic reviews of the literature between 1966 and 2007 [10, 11]. The most recent review [12] provided the most extensive overview. The three systematic reviews found that all psychotropic drug classes were associated with an increased risk of falling. The pooled odds ratios (ORs) ranged between 1.37 and 1.71 for antipsychotics, between 1.31 and 1.54 for sedatives and hypnotics and between 1.59 and 1.72 for antidepressants. It should be noted that these studies present the drugs by classes, whereas the specific drugs within these classes may have different chemical properties and may thus have different effects on the risk of falling.

Literature on the contribution of specific psychotropic drugs to fall frequency in older people is inconsistent. Only a few studies have investigated the fall risk of specific drugs. Two studies showed that the use of the sedative drug zolpidem is associated with an increased risk of falling, with an OR of between 2.51 and 4.37 [13, 14], which are both higher than the pooled estimates for the whole drug class of sedatives. An increased fall risk for individual drugs has been found for haloperidol (OR 6.09) [15]. Some studies did not show an increased risk of falling for the atypical antipsychotic drugs quetiapine and risperidone [16], and no significant fall risk was found for temazepam [17]. Given the lack of evidence of fall risks for specific drugs, treatment guidelines often report only those risks associated with the classes of psychotropic drugs and not possible differences between the drugs within these classes. Insight into the differences between specific drugs could raise awareness among physicians and contribute to decisions regarding the prescription of certain drugs. Moreover, we expect our exploratory analyses could lead to more focused follow-up studies with clear hypotheses to be tested. Therefore, this study examines the association between specific psychotropic drugs and fall incidents in nursing home residents.

\section{Methods}

\subsection{Study Design}

This was a retrospective cohort study. The cohort was identified using electronic pharmacy records from May 2012 until March 2015 and included somatic $(n=467)$ and psychogeriatric $(n=948)$ residents and former residents of long-stay wards in 22 nursing homes in the eastern part of the Netherlands. Short-stay residents ( $<60$ days) and physical rehabilitation residents were excluded from this study.

\subsection{Data Collection}

Medication records collected from the electronic prescription system included all prescribed drugs, their names and the start and stop dates as well as the sex and date of birth of each resident. Drugs prescribed on a pro re nata basis were excluded from analyses.

Electronic patient records provided patient admission and, if applicable, discharge dates. The incident report system provided records of all falls and other types of incidents. We did not use this system to retrieve information on the fall incidents, the patient's medical condition or the drug prescribed, because data are entered into this system as free text and were neither coded nor consistent.

\subsection{Data Analysis}

All collected data were anonymised by removing names and birthdates. All prescribed drugs were classified according to the anatomical therapeutic chemical (ATC) classification system [33]. Residents' prescriptions for identical drugs, and with adjacent stop and start dates, were combined into one prescription period.

A Cox proportional hazards model was used to investigate the magnitude of the association between the use of psychotropic drugs and the risk of falling during the first 60 days after the prescription start date [34]. Outcomes were calculated with a $95 \%$ confidence interval (CI). Crude hazard ratios (HRs) and adjusted hazard ratios (aHR) were calculated, with the age, sex, type of department (somatic or psychogeriatric), polypharmacy, and the use of other psychotropic drugs as covariates. The use of drugs from 
ATC classes N05A (antipsychotics), N05B (anxiolytics), N05C (hypnotics and sedatives), N06A (antidepressants) and N06D (anti-dementia drugs) were entered as separate covariates. The time to fall (60-day period) was compared between users and non-users of a specific drug or drug class. Users were entered into the survival analysis on the prescription start date. Non-users were entered into the reference group using a random date from their admission period. Additionally, possible differences in the effects of psychotropic drugs on fall incidents were also analysed for residents receiving psychogeriatric care and residents receiving somatic care by analysing the two groups separately. The model was adjusted for age, sex, polypharmacy, and the use of other psychotropic drugs as covariates.

All data were processed and analysed with Microsoft ${ }^{\circledR}$ Excel 2010, MySQL Workbench 6.2 and IBM SPSS Statistics 21.0.

\section{Results}

A total of 1415 residents were included, with a combined number of 698,567 patient-days and 3879 fall incidents. Within this cohort, 795 residents $(56.2 \%)$ experienced at least one fall during residence at the nursing home. This equated to 9.2 falls per 1000 patient-days for this subgroup and 5.6 falls per 1000 patient-days for the whole cohort. Table 1 presents the characteristics of the residents and the number of prescribed psychotropic drugs and fall incidents.

With an average use of $7.9( \pm 3.7)$ drugs, polypharmacy was highly prevalent among the cohort of 1415 residents. Antipsychotic drugs were prescribed to $42 \%$ of all residents at some point in the study period, and almost half of the residents $(47 \%)$ received at least one prescription for a sedative or hypnotic drug. The most commonly prescribed psychotropic drugs were temazepam (29\%), oxazepam (20\%) and haloperidol (19\%). On average, antidepressant drugs were used for 361 days (standard deviation [SD] 298), which was longer than any other psychotropic drug. The shortest intake duration was found for hypnotics and sedatives (average 175 days [SD 228]). Table 2 shows the magnitude of the association between the use of psychotropic drugs and fall incidents as analysed by the Cox proportional hazards model.

Looking at the five ATC classes of psychotropic drugs, an association was seen between fall incidents and the use of antipsychotics (aHR 1.49; 95\% CI 1.12-2.00), hypnotics and sedatives (aHR 1.51; 95\% CI 1.13-2.02), antidepressants (aHR 1.40; 95\% CI 1.02-1.85) and anxiolytics (aHR 1.39; 95\% CI 1.04-1.86). No association was found for anti-dementia drugs (aHR 1.32; 95\% CI 0.85-2.05).

Typical (aHR 1.40; 95\% CI 1.05-1.86) and atypical antipsychotics (aHR 1.73; 95\% CI 1.27-2.36) were both associated with fall incidents. However, differences were observed between drugs within these categories. The two most used typical antipsychotics were haloperidol and pipamperone; while haloperidol had an aHR of $1.54(95 \%$ CI 1.13-2.10), no clear association between the use of pipamperone and fall incidents was found (aHR 1.38; 95\% CI 0.91-2.10). Similar differences within drug categories were also visible for the two most used atypical antipsychotics, quetiapine (aHR 1.99; 95\% CI 1.39-2.85) and risperidone (aHR 1.31; 95\% CI 0.81-2.12). Within hypnotics and sedatives, an increased risk of falling was found for temazepam, zopiclone, zolpidem and melatonin but not for midazolam: the number of falls $(n=7)$ in midazolam users ( $n=158$ ) was lower than that for users of other drugs from this group.

As shown in Table 1, the incidence of falls for residents in psychogeriatric wards was higher than for those in somatic wards: 25 versus $5 \%$, respectively, fell more than four times during their admission. We performed additional analyses to investigate a possible difference in the magnitude of the risk of falling and the use of psychotropic drugs in somatic and psychogeriatric residents; the results are shown in Table 3.

Table 3 shows a correlation between the use of antipsychotics (both typical and atypical) and hypnotics and sedatives and fall incidents in psychogeriatric residents. No clear association between the use of any type of psychotropic drugs and fall incidents was found for somatic care residents.

\section{Discussion}

This study reports on the association between falls and several specific psychotropic drugs and psychotropic drug classes that are commonly prescribed for nursing home residents. The risk of falling appeared to increase with the use of both typical and atypical antipsychotics, hypnotics and sedatives, antidepressants and anxiolytics, with aHRs varying between 1.39 and 1.73. A link to falls was found with the use of the following specific drugs: zolpidem (aHR 2.35), melatonin (aHR 1.97), quetiapine (aHR 1.99), temazepam (aHR 1.96), zopiclone (aHR 1.81) and haloperidol (aHR 1.54). Other specific drugs, such as pipamperone, risperidone, midazolam, amitriptyline and citalopram did not show a clear association with falls. Antidementia drugs as a group also did not show any association with falls. The increased risks were especially observed in residents receiving psychogeriatric care, and falling is also much more prevalent among this group of residents than among somatic residents.

For the drug classes and three specific drugs (haloperidol, temazepam and zolpidem) that have been investigated 
Table 1 Characteristics of 1415 residents, their falling incidents and their drug prescription data from a total of 1912.6 patient years, observed between May 2012 and March 2015

\begin{tabular}{|c|c|c|c|}
\hline Characteristics & Total $(N=1415)$ & $\begin{array}{l}\text { Psychogeriatric residents } \\
(n=948[67 \%])\end{array}$ & $\begin{array}{l}\text { Somatic residents } \\
(n=467[33 \%])\end{array}$ \\
\hline $\mathrm{Age}^{\mathrm{a}}$ & $83.5( \pm 8.8)$ & & \\
\hline \multicolumn{4}{|l|}{ Sex } \\
\hline Female & $1000(71)$ & $687(72)$ & $313(67)$ \\
\hline Male & $415(29)$ & $261(28)$ & $154(33)$ \\
\hline Number of drugs used simultaneously & $7.9(3.7)$ & $7.2(3.4)$ & $9.1(3.8)$ \\
\hline \multicolumn{4}{|c|}{ Number of residents who received psychotropic drugs at least once in the study period } \\
\hline Antipsychotics & $594(42)$ & $480(51)$ & $114(25)$ \\
\hline Typical antipsychotics & $409(29)$ & $339(36)$ & $70(15)$ \\
\hline Haloperidol & $276(20)$ & $216(23)$ & $60(13)$ \\
\hline Pipamperone & $131(9)$ & $124(13)$ & $7(2)$ \\
\hline Atypical antipsychotics & $281(20)$ & $221(23)$ & $60(13)$ \\
\hline Quetiapine & $141(10)$ & $114(12)$ & $27(6)$ \\
\hline Risperidone & $93(7)$ & $79(8)$ & $14(3)$ \\
\hline Anxiolytics & $438(31)$ & $330(35)$ & $108(23)$ \\
\hline Oxazepam & $281(20)$ & $223(24)$ & $58(13)$ \\
\hline Lorazepam & $150(11)$ & $117(12)$ & $33(7)$ \\
\hline Hypnotics and sedatives & $664(47)$ & $443(47)$ & $221(47)$ \\
\hline Temazepam & $416(29)$ & $262(28)$ & $154(33)$ \\
\hline Midazolam & $158(11)$ & $116(12)$ & $42(9)$ \\
\hline Zopiclone & $121(9)$ & $90(10)$ & $31(7)$ \\
\hline Zolpidem & $94(7)$ & $70(7)$ & $24(5)$ \\
\hline Melatonin & $102(7)$ & $76(8)$ & $26(6)$ \\
\hline Antidepressants & $502(36)$ & $339(36)$ & $163(35)$ \\
\hline Amitriptyline & $108(8)$ & $68(7)$ & $40(9)$ \\
\hline Citalopram & $177(13)$ & $135(14)$ & $42(9)$ \\
\hline Anti-dementia drugs & $110(8)$ & $97(10)$ & $13(3)$ \\
\hline \multicolumn{4}{|l|}{ Average number of days used } \\
\hline Antipsychotics & $227 \pm 255$ & $230 \pm 256$ & $211 \pm 247$ \\
\hline Anxiolytics & $216 \pm 259$ & $217 \pm 258$ & $206 \pm 270$ \\
\hline Hypnotics and sedatives & $175 \pm 228$ & $174 \pm 227$ & $183 \pm 231$ \\
\hline Antidepressants & $361 \pm 298$ & $371 \pm 297$ & $303 \pm 295$ \\
\hline Anti-dementia drugs & $340 \pm 286$ & $346 \pm 286$ & $225 \pm 224$ \\
\hline \multicolumn{4}{|l|}{ Fall characteristics } \\
\hline No falls & $618(44)$ & $314(33)$ & $304(66)$ \\
\hline One fall & $255(18)$ & $187(20)$ & $68(15)$ \\
\hline Two falls & $131(9)$ & $98(10)$ & $33(7)$ \\
\hline Three falls & $83(6)$ & $68(7)$ & $15(3)$ \\
\hline Four falls & $65(5)$ & $44(5)$ & $21(5)$ \\
\hline More than four falls & $259(18)$ & $236(25)$ & $23(5)$ \\
\hline Falls/1000 patient-days & 5.6 & 6.7 & 2.7 \\
\hline
\end{tabular}

Data are presented as mean $\pm \mathrm{SD}$ or $N(\%)$

${ }^{a}$ Average age is calculated using the study inclusion date for those using antipsychotics

previously, the results of this study are mostly in line with other research. We confirmed an increased risk of falling for residents who received antipsychotics [12]. The most recent meta-analysis [12] found increased risks for both the hypnotic and sedative and the antidepressant drug classes, which we also observed.

In contrast with other studies [17], we found temazepam, the most commonly used hypnotic, to be associated 
Table 2 Magnitude of the association between commonly prescribed psychotropic drugs, or drug classes, and falls among nursing home residents

\begin{tabular}{|c|c|c|c|c|c|}
\hline & ATC code ${ }^{a}$ & Number of users (falls) ${ }^{b}$ & Reference group (falls) ${ }^{b}$ & HR $(95 \%$ CI $)$ & $\operatorname{aHR}^{\mathrm{c}}(95 \% \mathrm{CI})$ \\
\hline Antipsychotics & N05A & $594(110)$ & $821(89)$ & $1.79(1.35-2.37)$ & $1.49(1.12-2.00)$ \\
\hline Typical antipsychotics & & $412(82)$ & $1003(126)$ & $1.68(1.27-2.22)$ & $1.40(1.05-1.86)$ \\
\hline Haloperidol & N05AD01 & $275(57)$ & $1140(153)$ & $1.64(1.21-2.22)$ & $1.54(1.13-2.10)$ \\
\hline Pipamperone & N05AD05 & $131(27)$ & $1284(184)$ & $1.51(1.01-2.26)$ & $1.38(0.91-2.10)$ \\
\hline Atypical antipsychotics & & $281(60)$ & $1134(141)$ & $1.82(1.35-2.46)$ & $1.73(1.27-2.36)$ \\
\hline Quetiapine & N05AH04 & $141(39)$ & $1274(167)$ & $2.35(1.66-3.33)$ & $1.99(1.39-2.85)$ \\
\hline Risperidone & N05AX08 & $93(19)$ & $1322(185)$ & $1.49(0.93-2.39)$ & $1.31(0.81-2.12)$ \\
\hline Anxiolytics & N05B & $438(85)$ & $977(121)$ & $1.64(1.24-2.17)$ & $1.39(1.04-1.86)$ \\
\hline Oxazepam & N05BA04 & $282(55)$ & $1133(153)$ & $1.49(1.10-2.04)$ & $1.41(1.03-1.92)$ \\
\hline Lorazepam & N05BA06 & $150(39)$ & $1265(178)$ & $2.02(1.43-2.87)$ & $1.53(1.07-2.20)$ \\
\hline Hypnotics and sedatives & N05C & $664(111)$ & $751(82)$ & $1.59(1.20-2.12)$ & $1.51(1.13-2.02)$ \\
\hline Temazepam & N05CD07 & $416(90)$ & $999(127)$ & $1.86(1.42-2.43)$ & $1.96(1.49-2.58)$ \\
\hline Midazolam & N05CD08 & $158(7)$ & $1257(183)$ & $1.47(0.68-3.16)$ & $1.08(0.49-2.36)$ \\
\hline Zopiclone & N05CF01 & $121(33)$ & $1294(181)$ & $2.21(1.54-3.19)$ & $1.81(1.24-2.64)$ \\
\hline Zolpidem & N05CF02 & $94(32)$ & $1321(186)$ & $2.79(1.92-4.07)$ & $2.35(1.61-3.44)$ \\
\hline Melatonin & N05CH01 & $102(31)$ & $1313(184)$ & $2.45(1.68-3.59)$ & $1.97(1.33-2.90)$ \\
\hline Antidepressants & N06A & $502(89)$ & $913(126)$ & $1.33(1.01-1.74)$ & $1.40(1.06-1.85)$ \\
\hline Amitriptyline & N06AA09 & $108(20)$ & 1307 (188) & $1.35(0.85-2.13)$ & $1.49(0.93-2.38)$ \\
\hline Citalopram & N06AB04 & $177(37)$ & $1238(182)$ & $1.47(1.04-2.10)$ & $1.29(0.89-1.85)$ \\
\hline Anti-dementia drugs & N06D & $110(23)$ & $1305(190)$ & $1.42(0.92-2.18)$ & $1.32(0.85-2.05)$ \\
\hline
\end{tabular}

Analyses based on a Cox proportional hazards model. Reference group: residents who have never used the investigated drug or drug class during the study period (May 2012-March 2015); the size of each reference group is 1415-number of users

$a H R$ adjusted hazard ratio, ATC anatomical therapeutic chemical, $C I$ confidence interval, $H R$ hazard ratio

${ }^{\text {a }}$ See the main text for the class indicated by each ATC code

b Number of residents who fell within 60 days of their inclusion date

c Adjusted for age, sex, polypharmacy, type of care and the use of other drugs from ATC classes N05A, N05B, N05C, N06A or N06D

with an increased risk of falling. These different results might be because other research was conducted in different health contexts, such as in hospital patients or communitydwelling subjects, whereas our study was conducted solely in nursing home residents. Additionally, an adjusted OR of 4.37 (95\% CI 3.34-5.76) was previously reported for zolpidem [14], which suggests a strong association between this drug and subsequent falling incidents; we found an increased risk (HR 2.35). Costa-Dias et al. [15] found an association between haloperidol and falls, with a reported OR of 6.09; we also found an increased risk, albeit smaller (aHR 1.54), for haloperidol. The difference might be due to adjusting for other drugs in variables in the current study and including nursing home residents instead of hospitalized patients. Furthermore, we calculated HRs instead of ORs, which might contribute to these quantitative differences. The advantage of HRs is that they represent an instantaneous risk rather than a cumulative risk and, as such, do not depend on the time period investigated.
When interpreting the results, one must consider that, in some instances, the association between a drug and falling could be due to a baseline difference in the risk of falling between users and non-users of the drug rather than the effect of the drug itself. For example, haloperidol is the most widely used neuroleptic for the treatment of acute delirium [18], and delirium itself is associated with a higher rate of falls. Data on the medical condition of residents in this study were unavailable, so we cannot completely rule out confounding by indication. Studies that did take this into account found an increased unadjusted risk for psychotropic drugs $[19,20]$ that declined after adjusting for comorbidities and health status. According to the STOPP criteria [9], quetiapine is an antipsychotic that can be prescribed for patients with parkinsonism since it is regarded as having fewer negative effects on motor control [21]. Therefore, the results for quetiapine might be confounded because physicians may prescribe it for residents who are already prone to falling. The relatively high aHR 
Table 3 Association between the use of psychotropic drugs and falls among somatic and psychogeriatric nursing home residents

\begin{tabular}{|c|c|c|c|c|c|c|c|}
\hline \multirow[t]{2}{*}{ ATC code } & & \multicolumn{3}{|l|}{ Somatic residents } & \multicolumn{3}{|c|}{ Psychogeriatric residents } \\
\hline & & 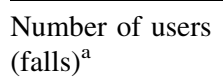 & 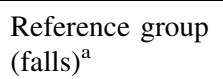 & $\mathrm{aHR}^{\mathrm{b}}(95 \% \mathrm{CI})$ & 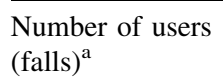 & 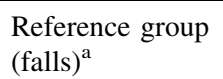 & $\mathrm{aHR}^{\mathrm{b}}(95 \% \mathrm{CI})$ \\
\hline Antipsychotics & N05A & $114(11)$ & $353(27)$ & $1.20(0.59-2.46)$ & $480(99)$ & $468(62)$ & $1.54(1.11-2.13)$ \\
\hline $\begin{array}{l}\text { Typical } \\
\text { antipsychotics }\end{array}$ & & $71(5)$ & $396(31)$ & $0.79(0.31-2.06)$ & $341(78)$ & $607(95)$ & $1.49(1.10-2.02)$ \\
\hline $\begin{array}{l}\text { Atypical } \\
\text { antipsychotics }\end{array}$ & & $60(7)$ & 407 (32) & $1.65(0.72-3.78)$ & $221(53)$ & 727 (109) & $1.73(1.24-2.42)$ \\
\hline Anxiolytics & N05B & $108(13)$ & $359(26)$ & $1.67(0.83-3.36)$ & $330(72)$ & $618(95)$ & $1.33(0.97-1.83)$ \\
\hline $\begin{array}{l}\text { Hypnotics and } \\
\text { sedatives }\end{array}$ & N05C & $221(19)$ & $246(16)$ & $1.40(0.71-2.80)$ & $443(92)$ & $505(66)$ & $1.60(1.16-2.21)$ \\
\hline Antidepressants & N06A & $163(19)$ & 304 (27) & $1.51(0.81-2.80)$ & $339(70)$ & $609(99)$ & $1.40(0.99-1.98)$ \\
\hline $\begin{array}{l}\text { Anti-Dementia } \\
\text { drugs }\end{array}$ & N06D & $13(2)$ & $454(40)$ & $2.2(0.51-9.73)$ & $97(21)$ & $851(150)$ & $1.25(0.79-2.00)$ \\
\hline
\end{tabular}

Analyses based on Cox proportional hazards model. Reference group: residents who have never used the investigated drug or drug class during the study period (May 2012-March 2015)

${ }^{a}$ Number of residents who fell who within 60 days after their analysis inclusion date

b Adjusted for age, sex, polypharmacy, type of care and the use of other drugs from ATC classes N05A, N05B, N05C, N06A or N06D (see the main text for the class indicated by each ATC code)

(1.99) could be influenced by the patient group rather than by the medicine. Depression is also known to increase the risk of falls [22], and antidepressants have the potential to impair gait, balance, and blood pressure regulation. However, the association between antidepressants and falls is unclear [7]. The most commonly prescribed antidepressant class in frail older adults is selective serotonin reuptake inhibitors (SSRIs) because they lack the side effects of traditional tricyclic antidepressants; however, this and other studies [22, 23] have found SSRIs to be associated with an increased risk of falling. Although a recent metaanalysis found that many observational studies indicated an association between SSRI use and falls, it also found that the evidence for SSRIs causing impairments in postural control in experimental studies such as RCTs was inconclusive [24, 25]. The STOPP criteria [9] recommend amitriptyline not be used as an antidepressant because it is associated with fall incidents. Therefore, the reason we did not find an association between fall risk and the use of amitriptylinemight be that it is prescribed in lower dosages for neuropathic pain and not as an antidepressant. We expect that confounding by indication also holds for the risk associated with melatonin. Physicians might be inclined to prescribe melatonin instead of hypnotic drugs such as temazepam to patients with an increased baseline fall risk, since melatonin is considered to have fewer side effects. However, it has been associated with an increased risk of fractures [26].

As expected, given the pharmacological properties, we did not find an increased risk of falls with anti-dementia drugs. These drugs are prescribed to prevent cognitive decline, for aggression in moderate to severe Alzheimer's disease (memantine) and for psychosis in Lewy Body dementia (cholinesterase inhibitors) [27]. The latter group has an increased baseline risk of falls [28]. Evidently, the potential risk of bias due to confounding by indication is not large enough to lead to a positive association with falls. Research indicates that differences exist within this drug class [29]; however, we chose not to split the anti-dementia drug group further because it was already small, and separate groups would have been too small for a regression analysis.

Also relevant is that we did not account for dosage in this study. However, other research suggests that the risk of falls rises as the dose of antipsychotics, anxiolytics, hypnotics or sedatives, and antidepressants increases and with the combined use of these drugs [30]. In our analysis, we adjusted for polypharmacy but did not adjust specifically for other drugs associated with an increased risk of falling. Additionally, the records of the fall incidents were dependent on nurses reporting the falls. Studies have indicated that falls that did not result in injuries were more often unreported than those that did [31]. This probably does not affect the relative risks of falling but might result in less accurate associations and larger confidence intervals. This type of information bias is likely to be nondifferential (i.e. the underestimation of falls is equal for users and nonusers of all drug classes), so this would not influence the HRs we found. Our data only included nursing home residents living in the eastern part of the 
Netherlands. Patient characteristics and the numbers receiving psychotropic drugs were comparable to other studies from the Netherlands [32]. Therefore, we believe our results are representative for the Netherlands.

While our results are in line with those of others in terms of the different drug classes, we cannot know whether the drug classes studied in the literature comprise the same drugs we studied. For example, while temazepam and midazolam were the most frequently prescribed drugs, they might not have been used as often in other studies performed at another time or in another country $[33,34]$. The personal or guideline-based treatment preferences of physicians may change over time, and new drugs may become available on the market $[35,36]$. Given the differential prescription rates of individual drugs, a fair comparison between the studies was difficult. This demonstrates the flaws inherent in studying whole drug classes and highlights the necessity of focusing on individual drugs. In this retrospective study, we were only able to adjust for a limited set of covariates (age, sex, polypharmacy, and the use of other psychotropic drugs). We were unable to adjust for comorbidity, functioning and mobility or rule out indication or prescription bias.

This study also has strengths that must be highlighted. The Cox model accounted for the type of care residents received (psychogeriatric or somatic) and the other possible confounders, such as age, sex, polypharmacy and the use of other psychotropic drugs. Furthermore, two of the three meta-analyses included studies from different health contexts, such as hospitals and nursing homes. The heterogeneity of these groups might have influenced results and increased variation. However, our results come from a more homogenous group [37]. Whereas somatic residents are usually characterized by serious physiological chronic disorders, psychogeriatric residents are characterized by serious mental or psychiatric disorders such as dementia. Consequently, comorbidities and functional status differ between these groups, and higher fall rates among psychogeriatric patients can also be attributed to the frequency of cognitive problems, behavioral problems and additional morbidity within this group. Therefore, we analyzed the two groups separately and expect less confounding.

Furthermore, this study included data for 1415 residents, with a combined 698,567 patient-days. The large amount of available data enabled us to analyze not only drug classes, such as antipsychotics, but also subclasses, such as typical and atypical antipsychotics and the most commonly used individual drugs.

Our study adds to the evidence regarding associations between psychotropic drugs and falls. Several drugs, such as haloperidol, quetiapine, temazepam, zolpidem and melatonin, seem to increase the risk of falls. However, these exploratory results should be further tested in a prospective study focussing on each of these individual drugs while also considering that fall incidents are multifactorial. Therefore, further study should also account for all potential confounders such as medical conditions (comorbidities, dementia severity, neuropsychiatric symptoms), mobility, additional medications (e.g. to determine the Drug Burden Index [38]) and indications for specific drugs.

Other commonly used antipsychotics, pipamperone and risperidone, were not associated with falls and therefore seem to be safe or at least prescribed safely. When we analysed the data for somatic and psychogeriatric residents separately, we found no clear association between psychotropic drugs and falls in somatic residents. These results suggest that assessing the risk of psychotropic drugs by looking at the drug class alone is unjustified because the fall risk may depend not only on the drug and dosage but also on the severity of the disease (which influences overall risk of fall). Further research focusing on the effects of the individual drugs is needed to provide physicians with more data that are applicable in practice. Current fall-prevention programmes consider entire drug classes to be a risk factor, but our results suggest this needs to be reviewed. Additionally, future studies should look at particular combinations of psychotropic drugs associated with falls given that nursing home residents often use several psychotropic drugs at once.

\section{Compliance with Ethical Standards}

Conflict of interest Sarah Janus, Gezinus Reinders, Jeannette van Manen, Sytse Zuidema, and Maarten IJzerman have no conflicts of interest that may be directly relevant to the content of this article.

Funding No sources of funding were used to conduct this study or prepare this manuscript.

Open Access This article is distributed under the terms of the Creative Commons Attribution-NonCommercial 4.0 International License (http://creativecommons.org/licenses/by-nc/4.0/), which permits any noncommercial use, distribution, and reproduction in any medium, provided you give appropriate credit to the original author(s) and the source, provide a link to the Creative Commons license, and indicate if changes were made.

\section{References}

1. Rubenstein LZ. Falls in older people: epidemiology, risk factors and strategies for prevention. Age Ageing. 2006;35:37-41.

2. Tideiksaar R. Falling in old age (prevention and management). 2nd ed. New York: Springer; 2007.

3. World Health Organization. Ageing, and life course unit. WHO global report on falls prevention in older age. Geneva: WHO; 2008.

4. Evans JG. Fallers, non-fallers and Poisson. Age Ageing. 1990;19(4):268-9.

5. Akyol AD. Falls in the elderly: what can be done? Int Nurs Rev. 2007;54(2):191-6. 
6. Hartikainen S, Lonnroos E, Louhivuori K. Medication as a risk factor for falls: critical systematic review. J Gerontol A Biol Sci Med Sci. 2007;62(10):1172-81.

7. Hajjar ER, Cafiero AC, Hanlon JT. Polypharmacy in elderly patients. Am J Geriatr Pharmacother. 2007;5(4):345-51.

8. Verhoeven V, Hartmann ML, Wens J, et al. Happy pills in nursing homes in Belgium: a cohort study to determine prescribing patterns and relation to fall risk. J Clin Gerontol Geriatr. 2014;5(2):53-7.

9. Gallagher P, O'Mahony D. STOPP (Screening Tool of Older Persons' potentially inappropriate Prescriptions): application to acutely ill elderly patients and comparison with Beers' criteria. Age Ageing. 2008;37(6):673-9.

10. Leipzig RM, Cumming RG, Tinetti ME. Drugs and falls in older people: a systematic review and meta-analysis: I. Psychotropic drugs. J Am Geriatr Soc. 1999;47(1):30-9.

11. Woolcott JC, Richardson KJ, Wiens MO, et al. Meta-analysis of the impact of 9 medication classes on falls in elderly persons. Arch Intern Med. 2009;169(21):1952-60.

12. Bloch F, Thibaud M, Dugué B, et al. Psychotropic drugs and falls in the elderly people: updated literature review and meta-analysis. J Aging Health. 2011;23(2):329-46. doi:10.1177/ 0898264310381277.

13. Rhalimi M, Helou R, Jaecker P. Medication use and increased risk of falls in hospitalized elderly patients. Drugs Aging. 2009;26(10):847-52

14. Kolla BP, Lovely JK, Mansukhani MP, et al. Zolpidem is independently associated with increased risk of inpatient falls. J Hosp Med. 2013;8(1):1-6.

15. Costa-Dias MJ, Oliveira AS, Martins T, et al. Medication fall risk in old hospitalized patients: a retrospective study. Nurse Educ Today. 2014;34(2):171-6.

16. Chatterjee S, Chen H, Johnson ML, et al. Risk of falls and fractures in older adults using atypical antipsychotic agents: a propensity score-adjusted, retrospective cohort study. Am J Geriatr Pharmacother. 2012;10(2):83-94.

17. Sylvestre M-P, Abrahamowicz M, Čapek R, et al. Assessing the cumulative effects of exposure to selected benzodiazepines on the risk of fall-related injuries in the elderly. Int Psychogeriatr. 2012;24(04):577-86.

18. Fong TG, Tulebaev SR, Inouye SK. Delirium in elderly adults: diagnosis, prevention and treatment. Nat Rev Neurol. 2009;5(4):210-20.

19. Landi F, Onder G, Cesari M, et al. Psychotropic medications and risk for falls among community-dwelling frail older people: an observational study. J Gerontol A Biol Sci Med Sci. 2005;60(5):622-6.

20. Hien LT, Cumming RG, Cameron ID, et al. Atypical antipsychotic medications and risk of falls in residents of aged care facilities. J Am Geriatr Soc. 2005;53(8):1290-5.

21. Sultzer DL, Davis SM, Tariot PN, et al. Clinical symptom responses to atypical antipsychotic medications in Alzheimer's disease: phase 1 outcomes from the CATIE-AD effectiveness trial. Am J Psychiatry. 2008;165(7):844-54.

22. Quach L, Penninx BW, Jones RN, et al. Depression, antidepressants, and falls among community-dwelling elderly people: the MOBILIZE Boston study. J Gerontol A Biol Sci Med Sci. 2013;68(12):1575-81.

23. Gribbin J, Hubbard R, Gladman J, et al. Serotonin-norepinephrine reuptake inhibitor antidepressants and the risk of falls in older people: case-control and case-series analysis of a large UK primary care database. Drugs Aging. 2011;28(11):895-902.

24. Gebara MA, Lipsey KL, Karp JF, et al. Cause or effect? Selective serotonin reuptake inhibitors and falls in older adults: a systematic review. Am J Geriatr Psychiatry. 2015;23(10):1016-28.

25. de Groot MH, van Campen JP, Moek MA, et al. The effects of fall-risk-increasing drugs on postural control: a literature review. Drugs Aging. 2013;30(11):901-20.

26. Frisher M, Gibbons N, Bashford J, et al. Melatonin, hypnotics and their association with fracture: a matched cohort study. Age Ageing. 2016;45(6):801-6.

27. O’Brien JT, Burns A, BAP Dementia Consensus Group. Clinical practice with anti-dementia drugs: a revised (second) consensus statement from the British Association for Psychopharmacology. J Psychopharmacol. 2011;25(8):997-1019.

28. Ballard CG, Shaw F, Lowery K, et al. The prevalence, assessment and associations of falls in dementia with Lewy bodies and Alzheimer's disease. Dement Geriatr Cogn Disord. 1999;10(2):97-103.

29. Olazarán J, Valle D, Serra JA, et al. Psychotropic medications and falls in nursing homes: a cross-sectional study. J Am Med Dir Assoc. 2013;14(3):213-7.

30. Sterke CS, Beeck EF, Velde N, et al. New insights: dose-response relationship between psychotropic drugs and falls: a study in nursing home residents with dementia. J Clin Pharmacol. 2012;52(6):947-55.

31. Evans SM, Berry JG, Smith BJ, et al. Attitudes and barriers to incident reporting: a collaborative hospital study. Qual Saf Health Care. 2006;15(1):39-43.

32. Taxis $\mathrm{K}$, Kochen $\mathrm{S}$, Wouters $\mathrm{H}$, et al. Cross-national comparison of medication use in Australian and Dutch nursing homes. Age Ageing. doi:10.1093/ageing/afw218. (Epub 14 Dec 2016).

33. Gallini A, Andrieu S, Donohue JM, et al. Trends in use of antipsychotics in elderly patients with dementia: impact of national safety warnings. Eur Neuropsychopharmacol. 2014;24(1):95-104.

34. Guthrie B, Clark SA, Reynish EL, et al. Differential impact of two risk communications on antipsychotic prescribing to people with dementia in Scotland: segmented regression time series analysis 2001-2011. PLoS One. 2013;8(7):e68976.

35. Donohue J, O'Malley AJ, Horvitz-Lennon M, et al. Changes in physician antipsychotic prescribing preferences, 2002-2007. Psychiatr Serv. 2014;65(3):315-22.

36. Hamann J, Kolbe G, Cohen R, et al. How do psychiatrists choose among different antipsychotics? Eur J Clin Pharmacol. 2005;61(11):851-4.

37. Verbeek H, Zwakhalen SM, Van Rossum E, et al. Small-scale, homelike facilities versus regular psychogeriatric nursing home wards: a cross-sectional study into residents' characteristics. BMC Health Serv Res. 2010;10:30.

38. Hilmer SN, Mager DE, Simonsick EM, et al. A drug burden index to define the functional burden of medications in older people. Arch Intern Med. 2007;167(8):781-7. 